Europ. Surg. Res. 1973;5:475-476

\title{
Authors Index
}

Aardal, Nils Petter 362 Abouna, George M. 390,

424 Ackern, K. van 414 Ahonen, J. 228, 259 Alho, Antti 333 Angelescu, N. 292 Aquino, C.J. 105 Aronsen, K. F. 268 Auranen, A. 228,259

Bénichoux, R. 292 Bismuth, H. 458 Bleser, F. 292 Boeckl, O. 11 Bornikoel, K. 202 Boss, j. H. 348 Bouveng, R. 146 Bowes, J. B. 21 Brendel, W. 162 Brown, Theodore H. 384 Bruckner, U. B. 414 Byström, Jan 401

Cascardo, Sergio 384 Castelfranchi, P. L. 105 Chalant, C. 37 Charlier, A. 37 Chaussy, Ch. 162 Chauvaud, S. 458 Collan, Yrjö 333 Condon, R. E. 64 Costello, M. 90 Cosyns, J. 37 Cotutiu, Coriolan 438

Dalton, R. G. 116 Davison, A. M. 116 Delin, N. Anders 194 Dent, D. 21 Deuster, J. E, 414

Dishon, T. 348 Durst, A. 348

Egenberg, Karl Edvard 3 Eriksson, Ejnar 401

Fara, J. 152 Fiore, Rosa 384 Francavilla, Antonio 384 Franco, D. 458

Gams, E. 202 Genell, Sven 321 Gentili, B. 129 Grange, D. 458 Groth, Carl G. 384

Hägerstrand, I. 268 Hagl, S. 202 Haglund, U. 152 Hahn, C. 37 Halvor^en, Jan F. 454 Hammer, C. 162 Hauf, E. 37 Heideman, Mats 321 Hell, E. 11 Hendrickx, J. 105 Hinchliffe, A. 21 Hussein, M. A. F. 73 Hvitfelt, J. 228, 259

Illi, E. 1

Jeske, Arthur H. 424 Jirsch, D. W. 216

Kestens, P. J. 273 Koláï, L. 52 Kolodej, A. 216 Kort, W. J. 81, 373 Kowalewski, K. 216

Kreuzer, W. 233 Kromrey, Catherine 26 Kux, M. 301 Kvetñanský, R. 58

Lambotte, L. 273 Lameijer, L. D. F. 81 Largiadèr, F. 1 Laszcz, M. 11 Leandoer, Lennart 321 Lefevre, J. 37 Leinberger, H. 414 Leon, C. 135 Lillehei, R. C. 105 Ljungqvist, Ulf 321 Longo, T. 175 Lorenz, W. 11 Lucas, T. R. 90

MacDicken, I. 81 Maizza, M. 129 Marchal, C. 292 Marchetti, G. 175 Marsili, M. 169 Martino, C. Di 129 Massion, W. H. 301 Mazzoni, G. 129 Meisner, H. 202 Mendler, N. 202 Merlo, L. 175 Meter, S. W. van 64 Meyer, W. 105 Moore, Francis D. 245 Moriau, M. 273 Myking, Andreas O. 454

Najarian, J. S. 26 Németh, S. 58 Norberg, Karl-Axel 194 Nordén, J. G. 268 Noseda, V. 175

Authors Index

Aardal, Nils Petter 362 Abouna, George M. 390,

424 Ackern, K. van 414 Ahonen, J. 228, 259 Alho, Antti 333 Angelescu, N. 292 Aquino, C.J. 105 Aronsen, K. F. 268 Auranen, A. 228,259

Bénichoux, R. 292 Bismuth, H. 458 Bleser, F. 292 Boeckl, O. 11 Bornikoel, K. 202 Boss, J. H. 348 Bouveng, R. 146 Bowes, J. B. 21 Brendel, W. 162 Brown, Theodore H. 384 Bruckner, U. B. 414 Byström, Jan 401 
Cascardo, Sergio 384 Castelfranchi, P. L. 105 Chalant, C. 37 Charlier, A. 37 Chaussy, Ch. 162 Chauvaud, S. 458 Collan, Yrjö 333 Condon, R. E. 64 Costello, M. 90 Cosyns, J. 37 Cotutiu, Coriolan 438

Dalton, R. G. 116 Davison, A. M. 116 Delin, N. Anders 194 Dent, D. 21 Deuster, J. E, 414

Dishon, T. 348 Durst, A. 348

Egenberg, Karl Edvard 3 Eriksson, Ejnar 401

Fara, J. 152 Filore, Rosa 384 Francavilla, Antonio 384 Franco, D. 458

Gams, E. 202 Genell, Sven 321 Gentili, B. 129 Grange, D. 458 Groth, Carl G. 384

Hägerstrand, I. 268 Hagl, S. 202 Haglund, U. 152 Hahn, C. 37 Halvorten, Jan F. 454 Hammer, C. 162 Hauf, E. 37 Heideman, Mats 321 Hell, E. 11 Hendrickx, J. 105 Hinchliffe, A. 21 Hussein, M. A. F. 73 Hvitfelt, J. 228, 259

Illi, E. 1

Jeske, Arthur H. 424 Jirsch, D. W. 216

Kestens, P. J. 273 Kolár, L. 52 Kolodej, A. 216 Kort, W. J. 81, 373 Kowalewski, K. 216

Kxeuzer, W. 233 Kromrey, Catherine 26 Kux, M. 301 Kvetñanský, R. 58

Lambotte, L. 273 Lameijer, L. D. F. 81 Largịadèr, F. 1 Laszcz, M. 11 Leandoer, Lennart 321 Lefevre, j. 37 Leinnberger, H. 414 Leon, C. 135

Lillehei, R. C. 105 Ljungqvist, Ulf 321 Longo, T. 175 Lorenz, W. 11 Lucas, T. R. 90

MacDicken, 1. 81 Maizza, M. 129 Marchal, C. 292 Marchetti, G. 175 Marsili, M. 169 Martino, C. Di 129 Massion, W. H. 301 Mazzoni, G. 129 Meisner, H. 202 Mendler, N. 202 Merlo, L. 175 Meter, S. W. van 64 Meyer, W. 105 Moore, Francis D. 245 Moriau, M. 273 Myking, Andreas O. 454

Najarian, J. S. 26 Németh, S. 58 Norberg, Karl-Axel 194 Nordén, J. G. 268

Noseda, V. 175

476

Authors Index

Novelli, G. P. 169

Odensjö, Gunilla 194 Ohlsson, E. G. 268 Olszewski, Waldemar 311 Otte, J. B. 273 Ottavá, Ludmila 401

Palacios, G. 135 Palmer, D. B. 21 Parmeggiani, A. 90 Pätru, Adriana 438 Pãusescu, Exacustodian 438 Peacock, J. H. 21 Pellegrini, S. 129 Pettersson, S. 282 Pieraccioli, E. 169 Pissiotis, C. A. 64 Plucinski, Slawomir 311 Pollet, J. 37 Post, Joke van der 373 Pouleur, H. 37

Reimann, H. J. 11 Reneman, R. S. 52 Reventos, J. 135 Ring, J. 202 Rink, R. D. 186 Ronnevi, Lars-Olof 401 Rosenmann, R. 348 Rubin, R. 90 Ruiz, O. J. 105

Sawyer, P. N. 90 Schenk, W. G., jr. 233 Scherstén, T. 282 Schildt, B. 146 Schmidt Habelmann, P. 202 Schmier, J. 414 Schraut, W. 202 Schulz, L. S. 105 Sebening, F. 202 Seidel, W. 11 Shons, A. R. 26 Sörbo, B. 146

Squifflet, J. P. 273 Srinivasan, S. 90 Stanczewski, B. 90 Struck, E. 202 Svanes, Knut 362

Taylor, Paul 384 Tomescu, Elena 438 Tricot, P. 292

Uhlig, R. 11 Uhlschmid, G. 1

Vicens, B. 135

Vigas, M. 58

Weissenhofer, W. 465 Westbroek, D. L. 81 Wieberdink, J. 52

Zimmermann, G. 11 